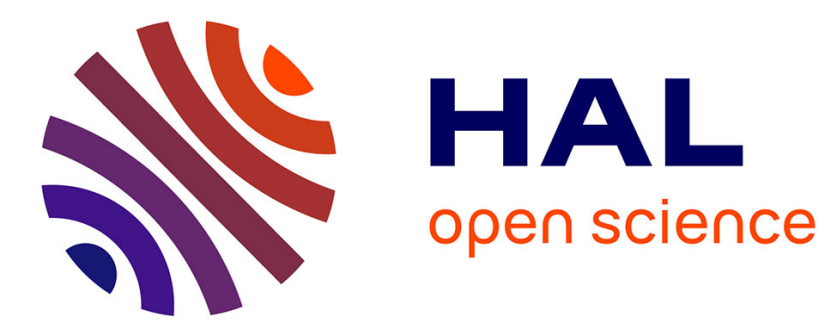

\title{
Enhanced absorption by nanostructured silicon
}

S. Bandiera, D. Jacob, T. Muller, François Marquier, Marine Laroche, Jean-Jacques Greffet

\section{To cite this version:}

S. Bandiera, D. Jacob, T. Muller, François Marquier, Marine Laroche, et al.. Enhanced absorption by nanostructured silicon. Applied Physics Letters, 2008, 93 (19), pp.193103. 10.1063/1.3021480 . hal-00574367

\section{HAL Id: hal-00574367 https://hal-iogs.archives-ouvertes.fr/hal-00574367}

Submitted on 16 Jul 2013

HAL is a multi-disciplinary open access archive for the deposit and dissemination of scientific research documents, whether they are published or not. The documents may come from teaching and research institutions in France or abroad, or from public or private research centers.
L'archive ouverte pluridisciplinaire HAL, est destinée au dépôt et à la diffusion de documents scientifiques de niveau recherche, publiés ou non, émanant des établissements d'enseignement et de recherche français ou étrangers, des laboratoires publics ou privés. 


\title{
Enhanced absorption by nanostructured silicon
}

\author{
S. Bandiera, D. Jacob, T. Muller, F. Marquier, M. Laroche, ${ }^{\text {a) }}$ and J.-J. Greffet \\ Laboratoire d'Energétique Moléculaire et Macroscopique, Combustion CNRS UPR 288, \\ Ecole Centrale Paris, Grande Voie des Vignes, F-92295 Châtenay-Malabry Cedex, France
}

(Received 21 July 2008; accepted 21 October 2008; published online 10 November 2008)

\begin{abstract}
Some applications such as ultrafast detectors or high efficiency photovoltaics require absorption by thin films. However, close to the bandgap, silicon absorbs very poorly. In this letter, we show that the absorption of a $100 \mathrm{~nm}$ slab can be as high as $50 \%$ in the range of wavelengths $700-830 \mathrm{~nm}$ when using a periodic structure properly designed. (C) 2008 American Institute of Physics.
\end{abstract}

[DOI: $10.1063 / 1.3021480$ ]

Emission and absorption efficiency of silicon for wavelengths close to its bandgap are very weak due to the indirect bandgap of this semiconductor. It is simple to analyze the different possibilities to address this issue. Absorption by a device is proportional to the integral over the device volume of $\operatorname{Im}(\epsilon)$, namely, the imaginary part of the dielectric constant and the square modulus of the electric field in the absorbing device

$$
P_{\mathrm{abs}} \propto \int_{V} \operatorname{Im}(\epsilon)|E|^{2} d V .
$$

The standard approach to enhance absorption is to increase the absorption volume. However, this is not desirable when dealing with electron-hole pairs that can recombine before being extracted. An alternative route is to increase the intrinsic losses given by $\operatorname{Im}(\epsilon)$. A lot of work in the field of material science has been devoted to modify the properties of the material. ${ }^{1}$ Finally, one can use optical resonators to enhance the local electromagnetic field $E$. It follows that complete absorption can be obtained with a given material and a small volume provided that the enhancement of the local field is large enough. In what follows, we use the name optical antennas for devices that play this role. Simple microcavities can be used in principle. Many applications in the field of resonant detectors have been reported along these lines. ${ }^{2}$ The limitation is that such structures are thick as they are made of a vertically grown stack of layers constituting the Bragg mirror.

However, solutions to increase the absorption of microstructured materials, taking advantage of other kind of resonances, have been studied. Total absorption by lamellar metallic gratings was demonstrated by Popov et al. ${ }^{3}$ Absorption of deep lamellar gratings of doped silicon exhibiting organ pipe modes was reported in Refs. 4 and 5. Also, absorption resonances can occur in dielectric antennas made of an infinite array of nanorods. ${ }^{6}$ Efficient optical antennas were also demonstrated for thermal emission. By excitation of surface plasmons on doped silicon, one could enhance thermal emission from shallow lamellar gratings and control its directivity. ${ }^{7}$ Similar properties were reported using the excitation of guided modes in periodically corrugated waveguides. ${ }^{8}$

In this letter, we show that a freestanding periodic membrane of $100 \mathrm{~nm}$, which is of the order of a tenth of the skin

${ }^{a)}$ Electronic mail: marine.laroche@em2c.ecp.fr. depth, of undoped silicon can absorb up to $50 \%$ of the incoming light at $\lambda=700-830 \mathrm{~nm}$. This is over one order of magnitude higher than the absorption factor of a homogeneous slab, which is on the order of $1 \%$ in this range of wavelengths. Two physical mechanisms will be emphasized, depending on the filling factor. For a weakly perturbed waveguide, the resonant absorption is shown to follow the dispersion relation of guided modes. For a small filling factor (strongly perturbed waveguide), we show that the physical mechanism differs from guided mode resonances.

As shown in Fig. 1, the system under study is a slab of silicon corrugated by a periodic array of infinite slits parallel to the $y$-axis. We denote $a$ as the period of the structure, $f$ is the filling factor, $t$ is the thickness of the slab, and $z$ is the normal axis. The incident electromagnetic plane wave is defined by its wave vector $\mathbf{k}$ and both incident angles $\theta$ and $\phi$. $\theta$ is the incident angle between the incident wavevector and the $z$-axis, whereas $\phi$ is the angle between the incident plane and the $x$-axis. The angle $\Psi$ between the electric field and the incident plane indicates the polarization. We denote TM polarization for $\Psi=0^{\circ}$ and TE polarization for $\Psi=90^{\circ}$. Neither the environment nor scale effects are discussed in this paper. Using the rigorous coupled wave analysis, ${ }^{9}$ we investigate the absorption properties in the visible-near infrared region of a freestanding membrane made of silicon. We have chosen to study the properties of a film of a given period $a$ $=0.54 \mu \mathrm{m}$ and thickness $t=0.1085 \mu \mathrm{m}$.

Let us first consider a weakly perturbed waveguide with high filling factor $f=0.95$. Figure 2 (a) displays a polar representation of the absorption of the structure for a given wavelength $\lambda=830 \mathrm{~nm}$. On $x$-axis is the normalized projection of the incident wavevector $\mathbf{k}_{x}=\sin (\theta) \cos (\phi)$ and on

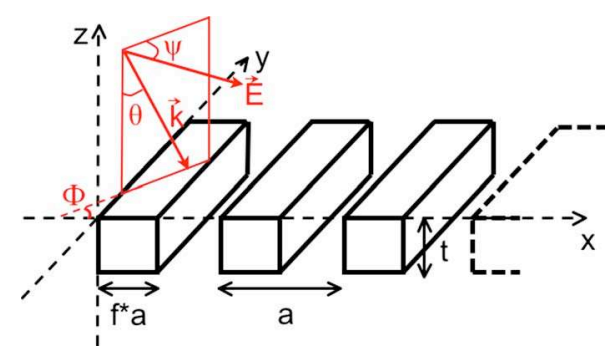

FIG. 1. (Color online) Schematic of the freestanding membrane of silicon with period $a=0.54 \mu \mathrm{m}$ and thickness $t=0.1085 \mu \mathrm{m}$. $\theta$ is the angle between the incident wavevector and the $z$-axis, and $\phi$ is the angle between the incident plane and the $x$-axis. We denote TM polarization for $\Psi=0^{\circ}$ and TE polarization for $\Psi=90^{\circ}$. 

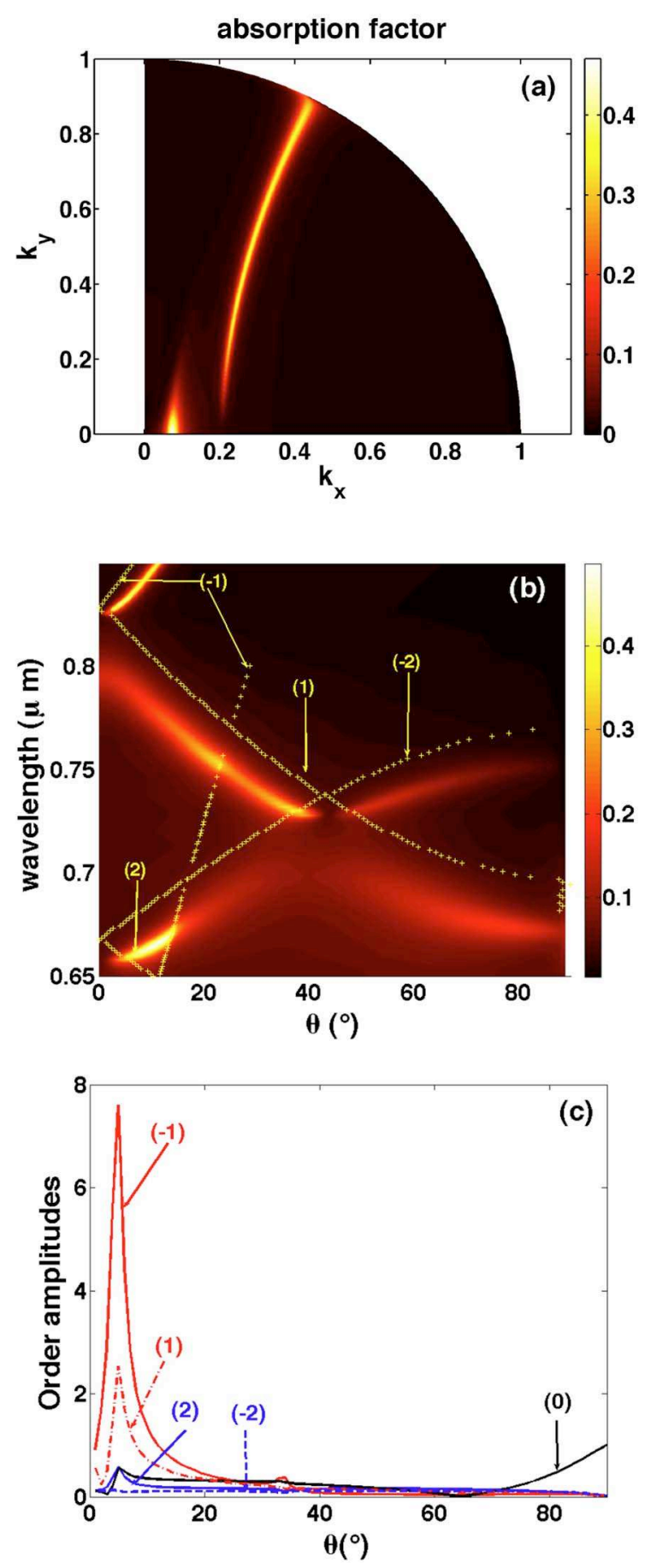

FIG. 2. (Color online) TM polarization. (a) Polar representation of the absorption factor for $\lambda=830 \mathrm{~nm}$ of a silicon grating with period $a$ $=0.54 \mu \mathrm{m}$, thickness $t=0.1085 \mu \mathrm{m}$, and filling factor $f=0.95$. (b) Dispersion relation of the unperturbed waveguide mode and absorption factor in the plane $(\theta, \lambda)$, respectively, represented by yellow markers + , and color scale from bright (0.5) to dark (0). (c) Amplitude of the $-1,+1,-2,+2$ diffracted orders (normalized to the amplitude of the incoming 0 order) vs the incident angle $\theta$ for $\phi=0^{\circ}, \lambda=830 \mathrm{~nm}$. At this wavelength, only the 0 order is propagating.

$y$-axis is the normalized projection of the incident wavevector $\mathbf{k}_{y}=\sin (\theta) \sin (\phi)$. The complex refractive index of the silicon is taken from Ref. 10. In Fig. 2(a), a bright line appears corresponding to a high absorption factor close to $50 \%$. Figure 2(b) shows the dispersion relation of the unperturbed waveguide mode and the absorption factor in TM polarization in the plane $(\theta, \lambda)$ for $\phi=0^{\circ}$. We observe that the region
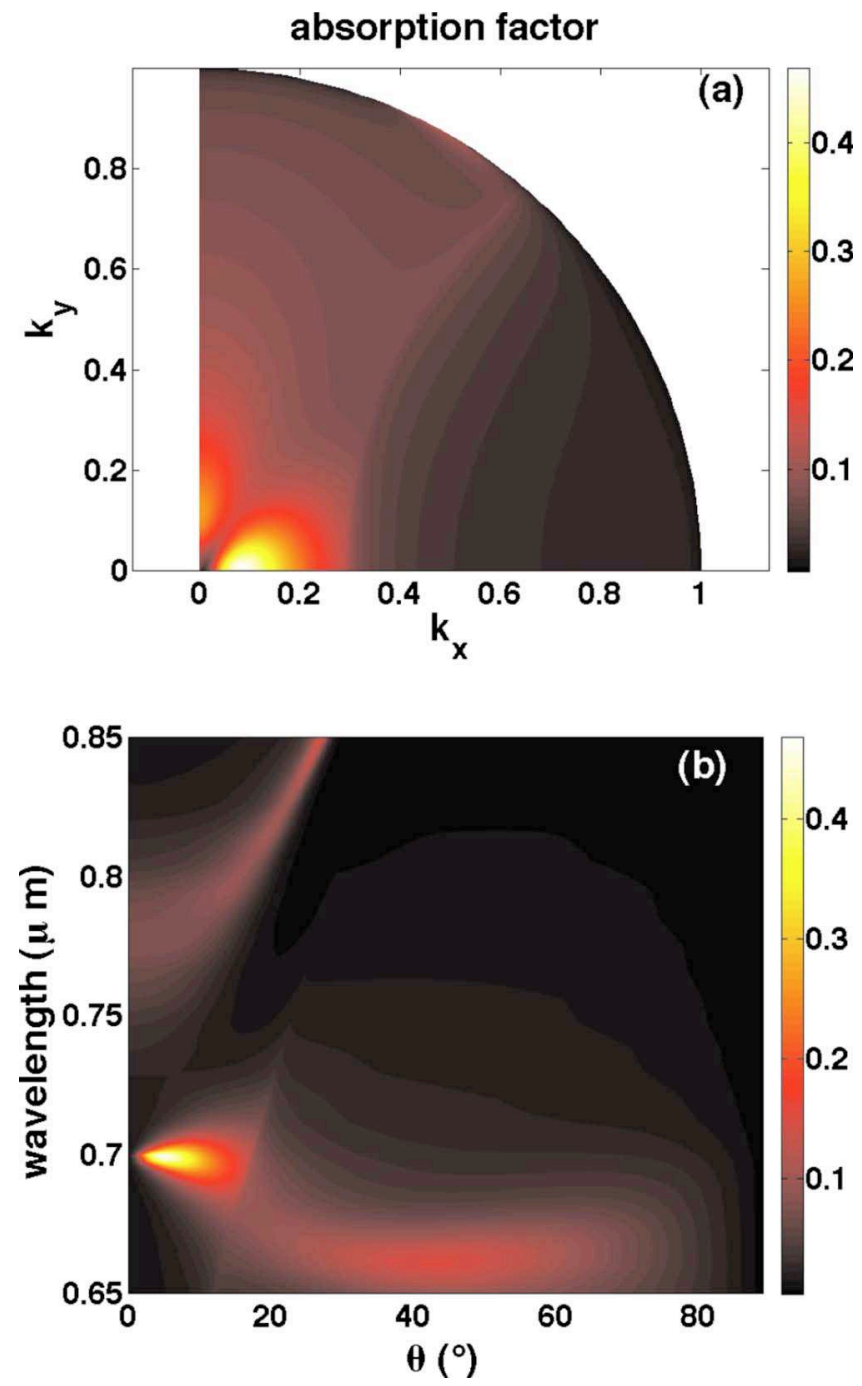

FIG. 3. (Color online) TM polarization. (a) Polar representation of the absorption factor for $\lambda=700 \mathrm{~nm}$ of a silicon grating with period $a$ $=0.54 \mu \mathrm{m}$, thickness $t=0.1085 \mu \mathrm{m}$, and filling factor $f=0.5$. (b) Absorption factor in the plane $(\theta, \lambda)$ represented by linear color scale from bright (0.5) to dark (0).

of high absorption coincides approximately with the dispersion relation of TM modes of the unperturbed waveguide (yellow + , calculated analytically) folded into the first Brillouin zone. Each line corresponds to an integer $p$ corresponding to the incoming wavevector $k=K_{W G}+p 2 \pi / a$, where $K_{W G}$ is the wavevector of the unperturbed guided mode. It is seen that the physical mechanism responsible for this high absorption is the resonant excitation of a guided mode coupled to the incoming propagating wave through the periodicity of the grating. The small discrepancy is due to the perturbation introduced by the slits in the dispersion relation of the guided modes. Figure 2(c) displays the amplitude of the diffracted orders at the illuminated interface of the grating versus the incident angle $\theta$ for $\phi=0^{\circ}$ or equivalently $k_{y}=0$, and $\lambda$ $=830 \mathrm{~nm}$. We see that the absorption peak coincides with the resonant excitation of the -1 order and then to the wavevector of the guided mode. This result confirms the interpretation of a coupling between the guided mode $(-1)$ of the grating and the incident wave. In summary, the absorption factor of a slightly perturbed silicon thin film can reach $50 \%$ at $830 \mathrm{~nm}$ by resonant coupling to the guided mode through the periodic array of slits. 


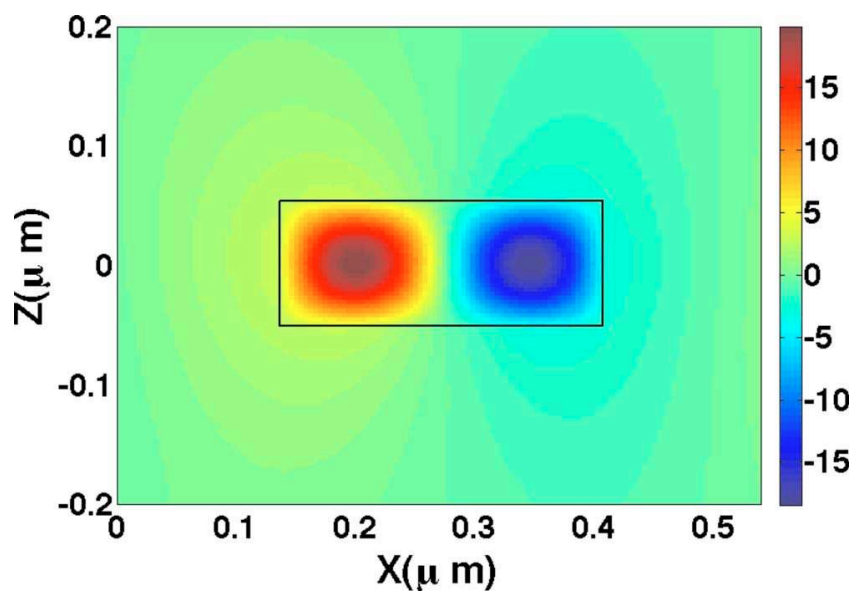

FIG. 4. (Color online) Map of the real part of the magnetic field at the absorption resonance $\lambda=700 \mathrm{~nm}, \theta=5.46^{\circ}$, and $\phi=0^{\circ}$. The dark line indicates the limit of the Si rod.

We now turn to the study of a strongly perturbed waveguide with filling factor $f=0.5$. Figure 3(a) displays the absorption factor in the normalized $\left(k_{x}, k_{y}\right)$ plane for a wavelength $\lambda=700 \mathrm{~nm}$. We observe a narrow and bright spot corresponding to a high and very directional absorption, close to $k_{x} \simeq 0.15$, corresponding to $\phi=0^{\circ}$ and $\theta=5^{\circ}$. This structure does not follow the dispersion relation of a guided mode for a homogeneous slab. This is not surprising for a filling factor $f=0.5$. Figure 3(b) represents the absorption factor in the plane $(\theta, \lambda)$ for $\phi=0^{\circ}$. The resonant absorption (bright spot around $\theta=5^{\circ}$ ) only exists in a very narrow range of incident angles and wavelengths. The field has also a much more complicated structure as the amplitudes of several evanescent diffracted orders (not shown) exhibit an amplification.

To understand the underlying physics of this resonance, we plot in Fig. 4 the map of the real part of the magnetic field at the resonance of absorption. We clearly see an enhancement of the field (normalized to the incident intensity) by a factor of 15 and a structure of an antisymmetric mode (with respect to the $x$-axis) inside the Si rod. This type of resonance was already discussed by Lalanne $e t$ al. in Ref. 11 in the case of a reflection resonance for a similar structure but with no losses. As discussed in the latter, this resonance can be interpreted in terms of Fabry-Pérot resonances of a weakly coupled antisymmetric Bloch mode propagating along the $z$-direction. The antisymmetry of the Bloch mode explains why the resonance cannot be excited in normal incidence. The Fabry-Pérot character is revealed by studying the dependence of the resonance wavelength as a function of

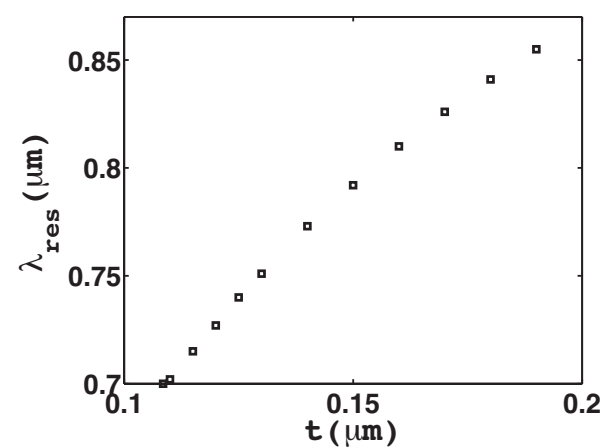

FIG. 5. Wavelength of the absorption resonance vs the thickness $t$ of the $\mathrm{Si}$ membrane. The angle of the absorption resonance (not shown) is always around $5^{\circ}$.

the thickness, as shown in Fig. 5. As expected, we find an almost linear dependence.

In conclusion, our results show that a resonant grating offers a very efficient way to improve the interaction of light and silicon close to the bandgap. With only $100 \mathrm{~nm}$ thick grating, the absorption can reach $50 \%$ in the spectral range $700-830 \mathrm{~nm}$. We have shown that depending on the filling factor of the grating, the absorption resonance was due to two different physical mechanisms. The first holds for a weakly corrugated waveguide and lies on the excitation of a guided mode. The second is due to the excitation of an antisymmetric mode which exhibits a vertical Fabry-Pérot resonance.

The authors acknowledge the ANR through the financial supports of the Institut Carnot of LETI and Thri-PV project, and the EU through the IP "Molecular Imaging" (LSHG-CT2003-503259). The authors thank Philippe Lalanne and Philippe Boucaud for useful discussions.

${ }^{1}$ S. Ossicini, L. Pavesi, and F. Priolo, Light Emitting Silicon for Microphotonics (Springer, Berlin, 2003).

${ }^{2}$ M. S. Unlu and S. Strite, J. Appl. Phys. 78, 607 (1995).

${ }^{3}$ E. Popov, L. Tsonev, and D. Maystre, Appl. Opt. 33, 5214 (1994).

${ }^{4}$ P. J. Hesketh, J. N. Zemel, and B. Gebhart, Nature (London) 324, 549 (1986).

${ }^{5}$ M. Auslender and S. Hava, Infrared Phys. Technol. 36, 1077 (1995).

${ }^{6}$ M. Laroche, S. Albaladejo, R. Gomez-Medina, and J. J. Saenz, Phys. Rev. B 74, 245422 (2006).

${ }^{7}$ F. Marquier, K. Joulain, J. P. Mulet, R. Carminati, and J.-J. Greffet, Opt. Commun. 237, 379 (2004).

${ }^{8}$ K. Joulain and A. Loizeau, J. Quant. Spectrosc. Radiat. Transf. 104, 208 (2007).

${ }^{9}$ N. Chateau and J. P. Hugonin, J. Opt. Soc. Am. A 11, 1321 (1994).

${ }^{10}$ E. D. Palik, Handbook of Optical Constants of Solids (Academic, San Diego, 1985).

${ }^{11}$ P. Lalanne, J. P. Hugonin, and P. Chavel, J. Lightwave Technol. 24, 2442 (2006) 